\title{
The Detection of Container Body Base on Multiple Features
}

\author{
CHEN Ziyi ${ }^{1}$, ZHENG Yinxi ${ }^{2}$, WANG Junhao ${ }^{2}$, WANG Lisheng ${ }^{1}$ \\ 1) Institute of Image Processing \& Pattern Recognition, Department of Automation, Shanghai Jiao \\ Tong University, Shanghai, 200240, China
}

\begin{abstract}
Since the 20th century, the global container transportation is developing very quickly. Unfortunately, although many container terminals have adopted modern management, the character recognition of containers still need the operation of manual read and manual record, which will not only cause the operation errors, but also slow the transportation speed. The character recognition system of container is the key to solve the problem, and in the system, the detection of the container body is a vital step. The traditional method for container detection is background subtraction, using an image with the container trunk to minus an image with no container trunks, then extracting the container body by the result. However, for the actual collected image, because of the factors of the low image resolution, the illumination change and the projection of the trunk, the brightness of origin background region will be changed a lot. If continue using traditional method, the extraction error may be made. Through the observation of the actual image, because the containers show obvious color features and texture features, a method based on HSV color model and texture features has been proposed. By the test, good results have been achieved.
\end{abstract}

Keywords: container detection; HSV color model; texture features;

\section{Introduction}

Since the second half of the 20th century, the global container transportation is becoming more and more perfect from technical equipment to the operation management, and the container transportation industry in our country is developing rapidly in recent decades. Unfortunately, although many container terminals have adopted the modern management, the character recognition still needs the operation modes of manual read and manual record. When the container carrier goes in and out of the wharf, human errors by the manual ways will inevitably exist, which greatly reduce the transportation speed of the container trucks. With the rapidly increase of the number of container, the existing facility and management level of the container terminal in our country can't meet the needs of the modern container transportation. Therefore, the use of the automatic container character recognition technology is the key to solve this problem. The automatic container character recognition system can recognize the character automatically, so the manual operation is not needed. The methods not only eliminate the mistake by manual operation, but also improve the efficiency.

The extraction of the container body in the image is a very important step of the automatic container character recognition system. The parking position of container carrier is changeable when it is photographed by the camera, so the position of the container body in the image is not sure. But the location of the container body is a key factor for the positioning of the container's character region. Thus, the location of the container body extraction is a vital step in the automatic container character recognition system.

The normal method to extract the container position is the method of background subtraction,

1 CHEN Ziyi

Institute of Image Processing \& Pattern Recognition, Department of Automation, Shanghai Jiao Tong University, Shanghai, 200240, China

e-mail:cy111sfncy@hotmail.com 
using an image with the container to minus a image without the container, then getting the R, G, B value of each pixel in the result image. If one of the $R, G, B$ value is greater than a certain threshold, the pixel is considered to be in the container region. Then, with certain morphological operation, the real actual container region can be got.

However, for the actual collected image, because of the factors of the low image resolution, the illumination change and the projection of the trunk, the brightness of origin background region will be changed a lot. Especially the factor of the projection of the trunk, which makes the bright part of the image be the shadow part. If we continue using the traditional method, a lot of background regions will be considered to be the container regions. Facing this situation, it is easy to make a wrong container extraction by the traditional method.

In the observation of the actual image, the container region shows obvious color characteristics. So, it is considered to use the color information of the image to detect the container region. HSV (hue, saturation and value) model is closer to people's experience and the perception of color than RGB model, so it is considered to use the HSV model for detection. Through experiment, for color container body, the method shows a very good detection result, but for the white container body, the result is not satisfactory. Through further observation, we have found that the outline of the container body also shows obvious texture feature, so we combine the method of texture feature and HSV model for the extraction of white container body.

In conclusion, a detection method of container body base on HSV color model and texture feature model has been proposed in this paper. The first step of this method is to judge the main body color. If the color is the partial to color, we use the HSV color model for extraction, and if the color is partial to white, we combine the texture feature model and HSV model for extraction.

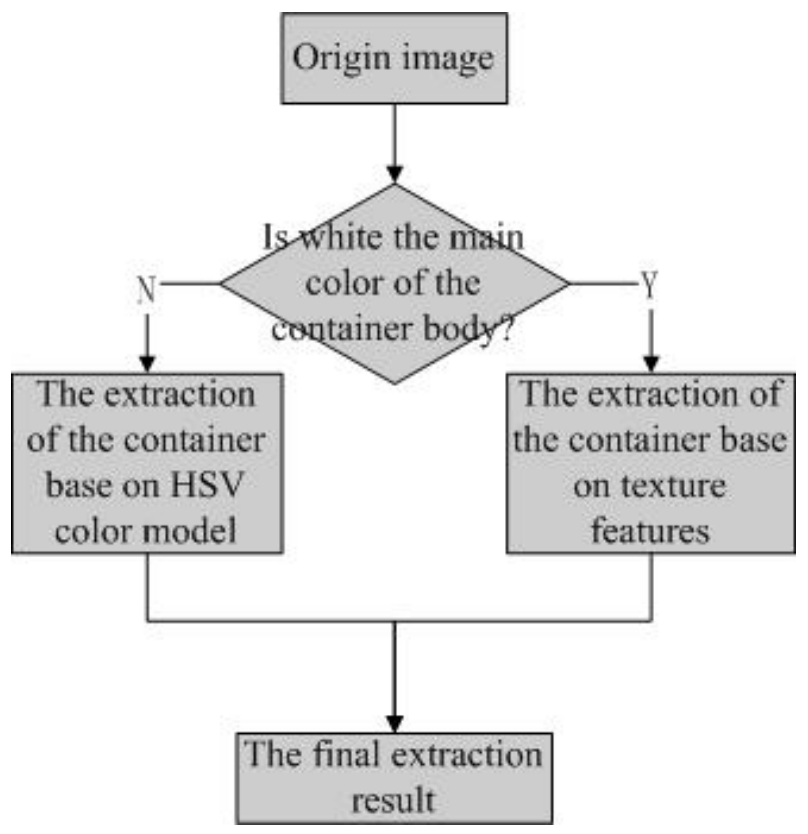

Fig.1.1 The main flow chart of the extraction of container body

\section{The Extraction Method for the Container of Color Body}

\subsection{The Color Model of HSV}

HSV model is one of the color systems, and it is used by people to select colors from the palette or color wheel (such as paint, ink, etc.). HSV represents hue, saturation, and value. The system is closer to people's experience and perception of color than RGB color system. 
The HSV color space model is corresponding to a conical subset of the cylindrical coordinate system, and the top of the cone represents $V=1$. It contains the three covers of RGB model that $R=1$, $\mathrm{G}=1$ and $\mathrm{B}=1$, and the color represented is lighter. $\mathrm{H}$ color is given by the rotate angle around $\mathrm{V}$ shaft. Red is corresponding to the angle of $0^{\circ}$, green is corresponds to the Angle of $120^{\circ}$, blue is corresponding to the angle of $240^{\circ}$. In the HSV color model, the difference of the complementary color of each color and itself is $180^{\circ}$. The $\mathrm{S}$ value is from 0 to 1 , so the radius of the cone top is 1 . HSV color space can be described by a conical space model.

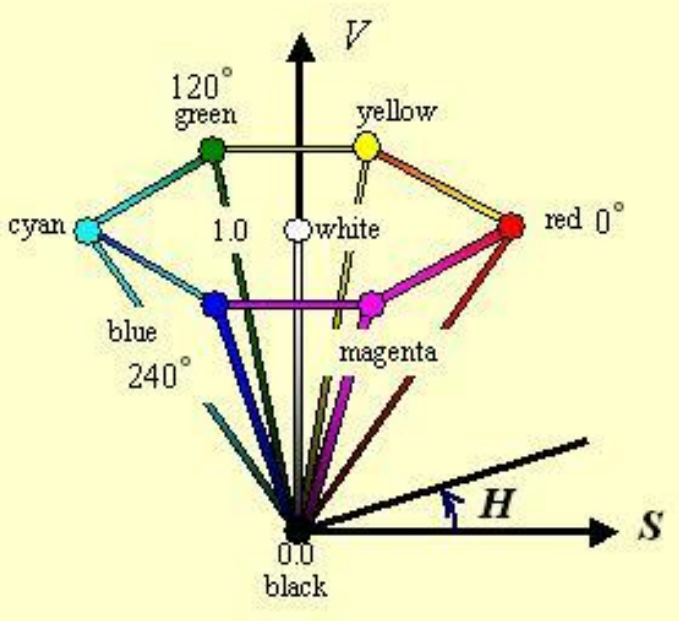

Fig.2.1 HSV color model

\subsection{The Judgement of Main Color of the Container Body and the Extraction of the Container Body}

The entire detection method mainly includes the color judgement of the container body and the extraction of the container region. Due to the low resolution of the origin image, the color information is not obvious, so we firstly reduce the origin image to $1 / 4$ of the origin size to highlight the color information.

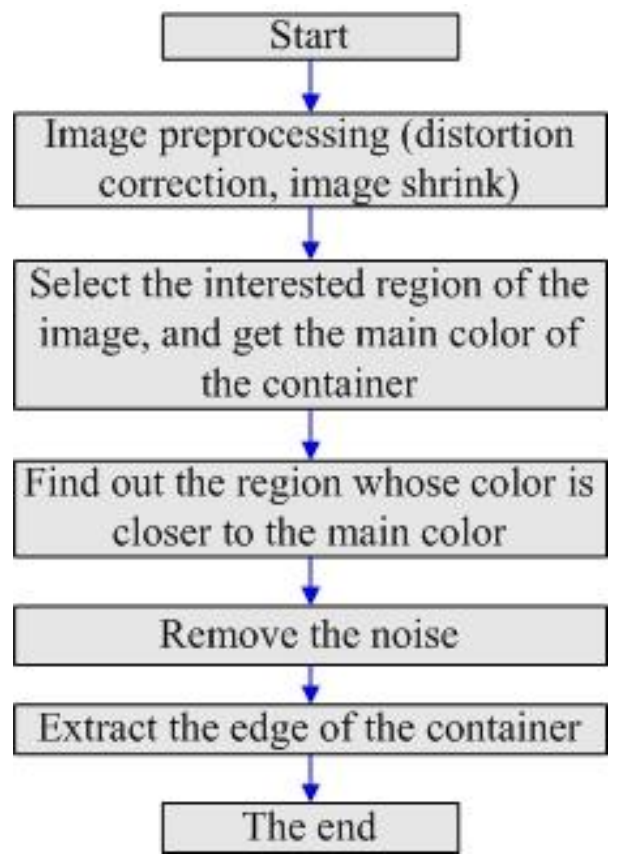

Fig.2.2 The flow chart of the container extraction of color body 
The detection method is as follows:

1) Choose the interested region of the shrink RGB image (according to the regulation, the center area of each image must be the container body area, so we set the central region to be our interested area), and transform it to a HSV image. As the following figures show:
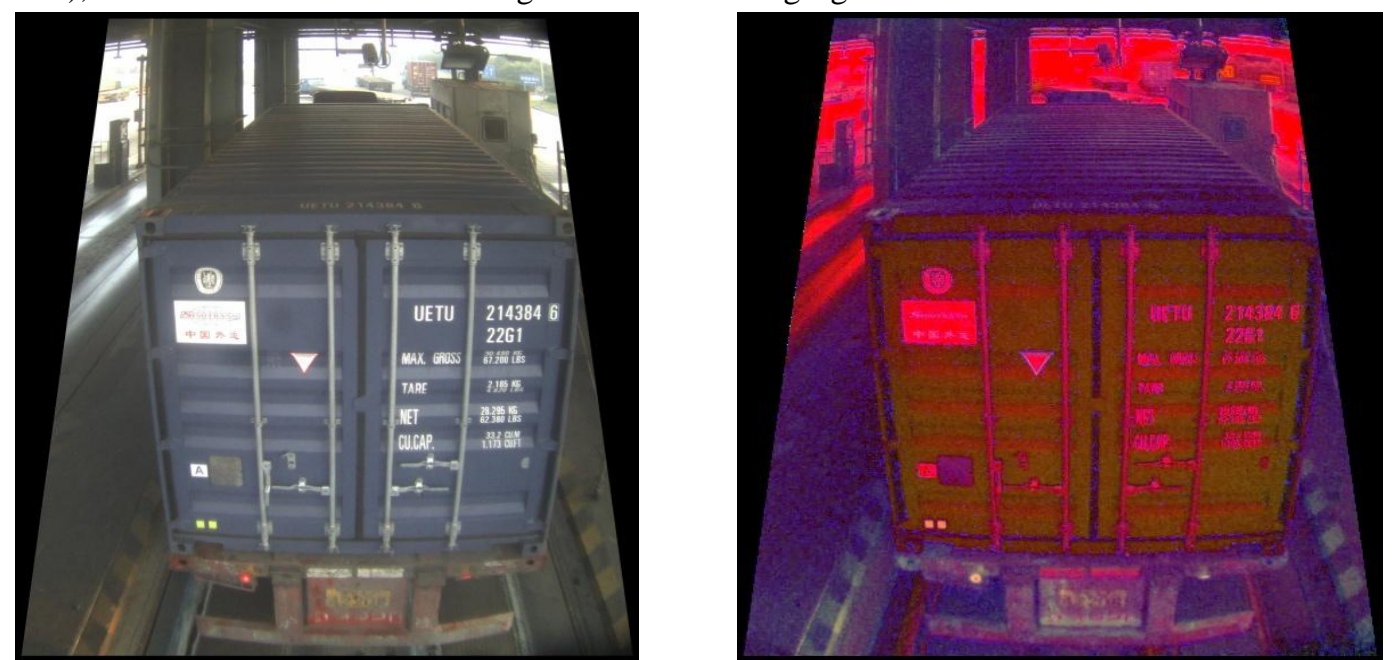

2) Create the histogram projection of $\mathrm{H}$ channel of the interested region, compute the highest $\mathrm{H}$ value in the histogram, then set it to $\operatorname{maxH}$, and it is considered that maxH is the main color of the container body. As the following figures show:
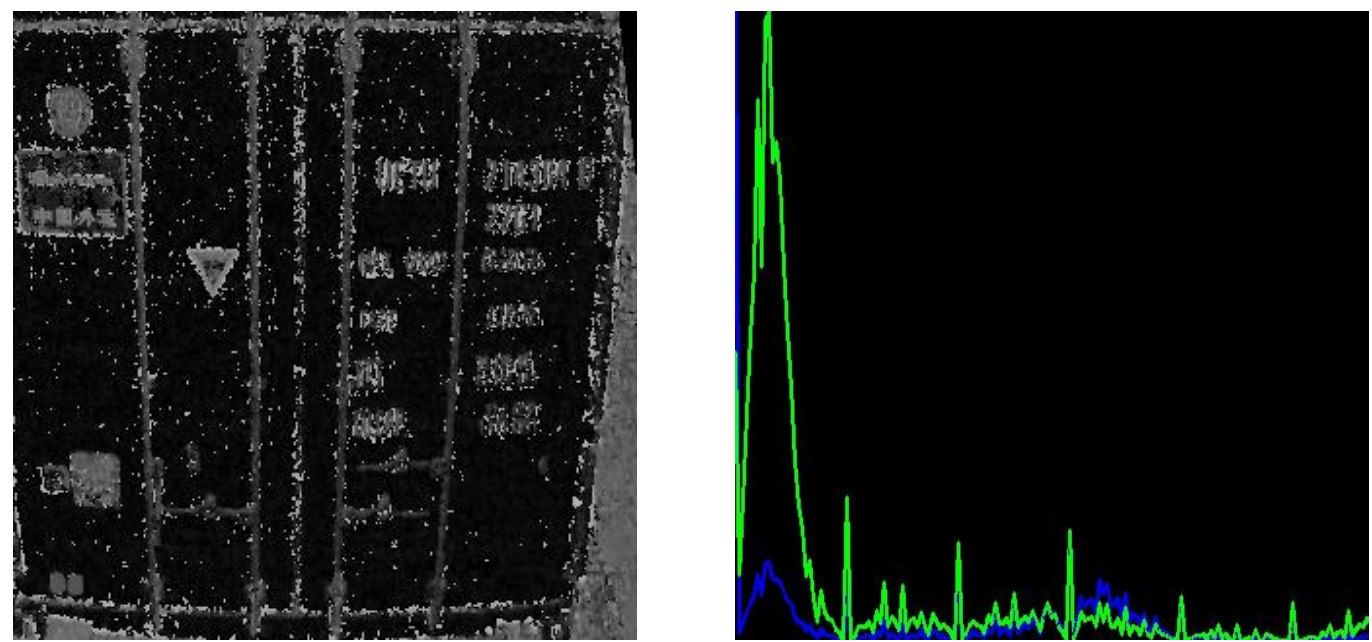

3) Find the region whose $\mathrm{H}$ value is between $[\operatorname{maxH}-\mathrm{x}, \operatorname{maxH}+\mathrm{x}]$ in the origin image $(\mathrm{x}$ is the acceptable error range). As the following figures show: 


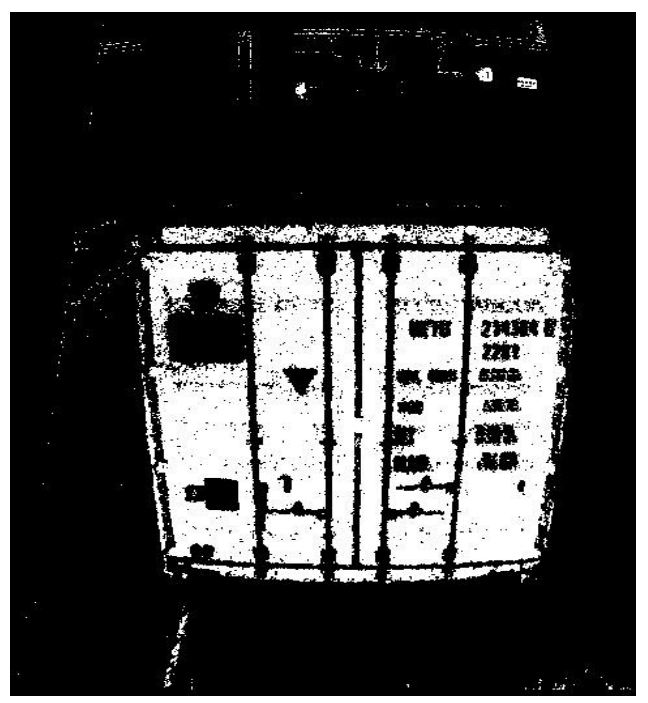

4) Remove the noise, and extract the rectangle border of the container body. As the following figures show:

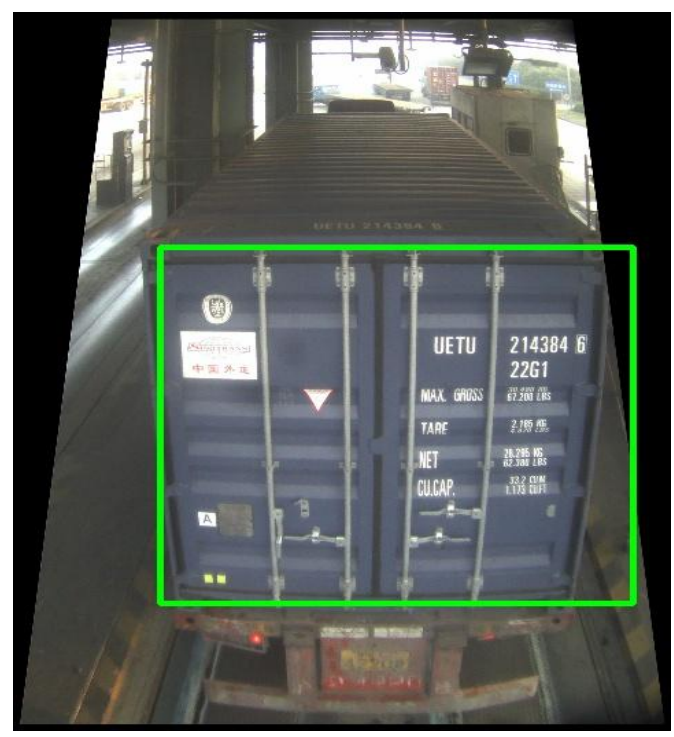

\section{The Container Body Detection Base on Texture Features}

\subsection{Sobel Filter and Scharr Filter}

The edge part of container body shows a very different texture feature than the background part, so we consider using the gradient on the edge of the container body to find the edge part of the container body.

Generally speaking, the most commonly used operation to express differential is Sobel differential operator. Sobel differential operator contains any order of differential and mixed partial derivative.

Sobel derivative has very good properties, that is, you can define any size of structure element, and the element can be constructed by fast and iterative way. Because the small element is more sensitive to noise, the big element has better approximation to the derivative. However, the use of big element will inevitably prolong the response time, and the use of small element will cause larger errors.

Scharr filter is as fast as Sobel filter, but its accuracy is higher, especially for the situation of small element. So here we use Scharr operator to filter the image. 


\subsection{The Method for Container Body Detection}

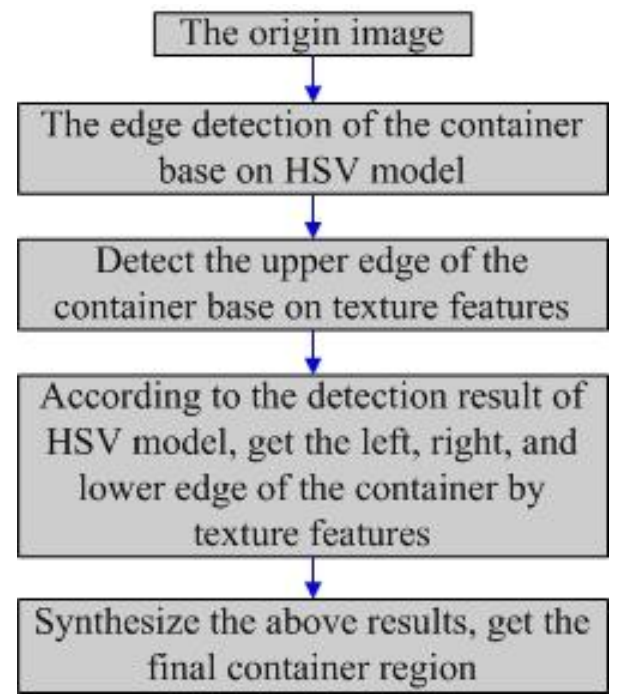

Fig.3.1 The flow chart of the method for the extraction of the container of white body

1) Use P1 to represent the origin image and use P2 to represent the region extracted by HSV model.

2) Extract the upper edge of the container body base on texture feature

Through the observation, there is a mutation of gradient between the background and the top of the container. The gradient of the top of the container is very small, and there is another mutation of gradient between the top of the container and the edge of the container body. The second mutation line is the actual upper edge of the container body.

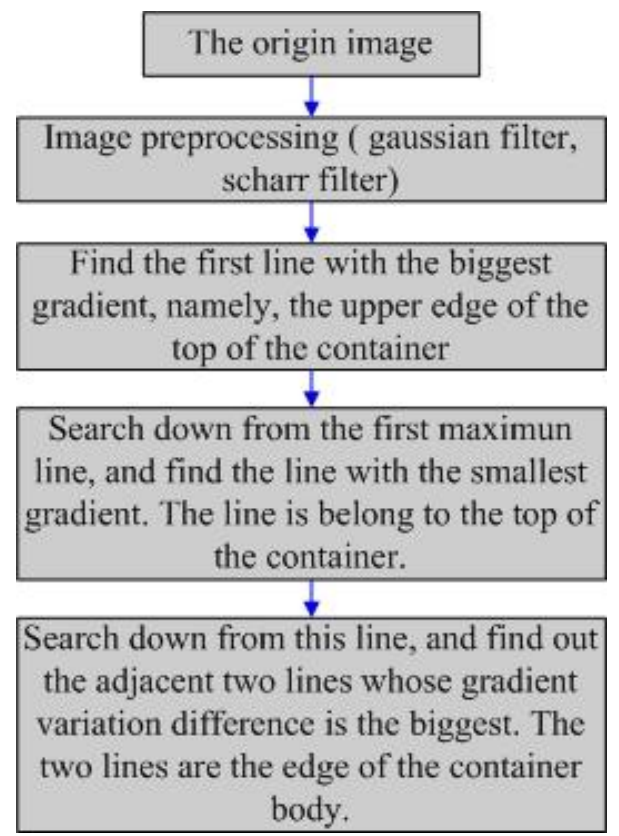

Fig.3.2 The flow chart of the extraction of the upper edge of the container base on texture features

3) The extraction of the left, right, and bottom edge of the container body

Through the observation, there is also a mutation of gradient between the background and each side edge of the container body. To eliminate the interference of the background region, we use $\mathrm{Y}$ to represent the left edge detected by HSV model, set the origin search the column of (Y-20), search the right direction of (cols/5), and the point where the gradient has changed biggest is the left border of the container body. 
The right edge can also be got by the same method.

At the same time, we use the result of HSV method to represent the lower edge. On one hand, the lower edge got by the HSV method is relatively accuracy, and the texture of the lower edge is relatively complex which is not suitable for detection. On the other hand, because of the ultimate goal of the container extraction is to position the character region of the image, so, the requirement of the accuracy rate of the lower edge is not very high.

4) Combine the two steps of 2) and 3), the final container region is got.

\section{The Test Results and Analysis}

In order to get better analysis results, we respectively discuss the color container and the white container.

\subsection{The Extraction Result of the Color Container}
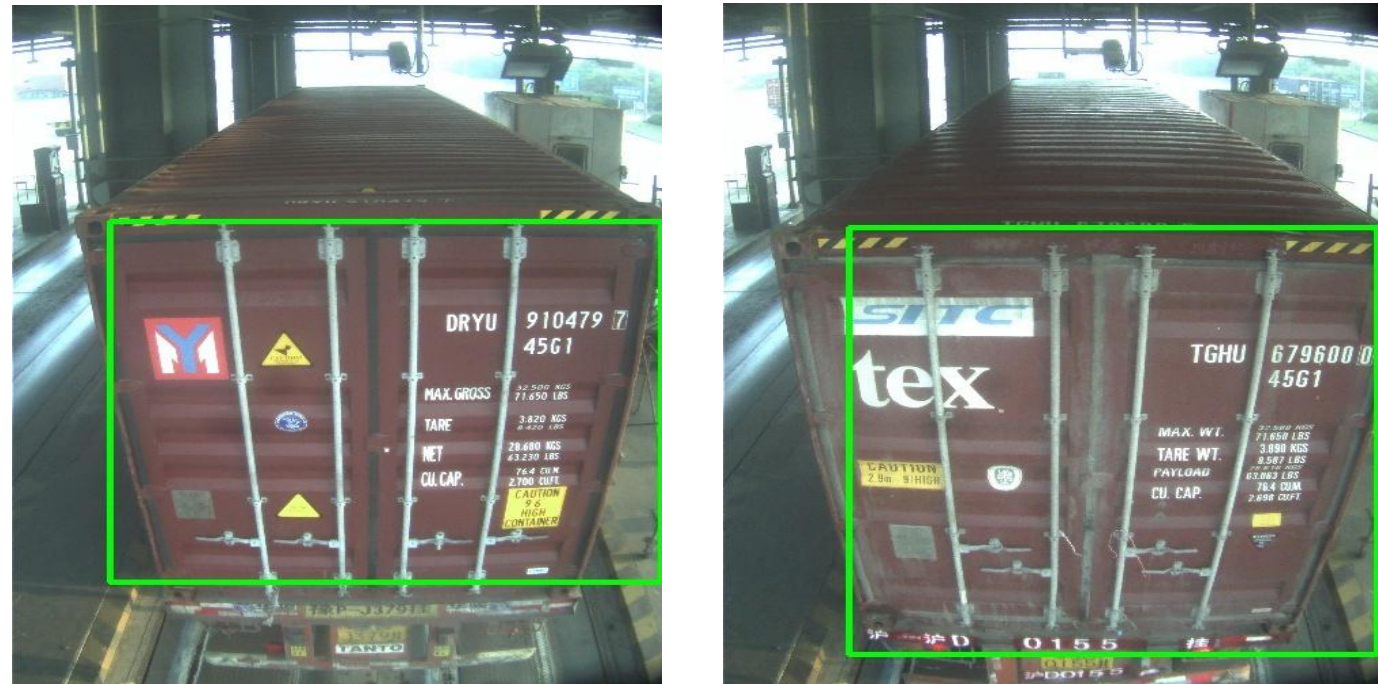

\subsection{The Extraction Result of the White Container}
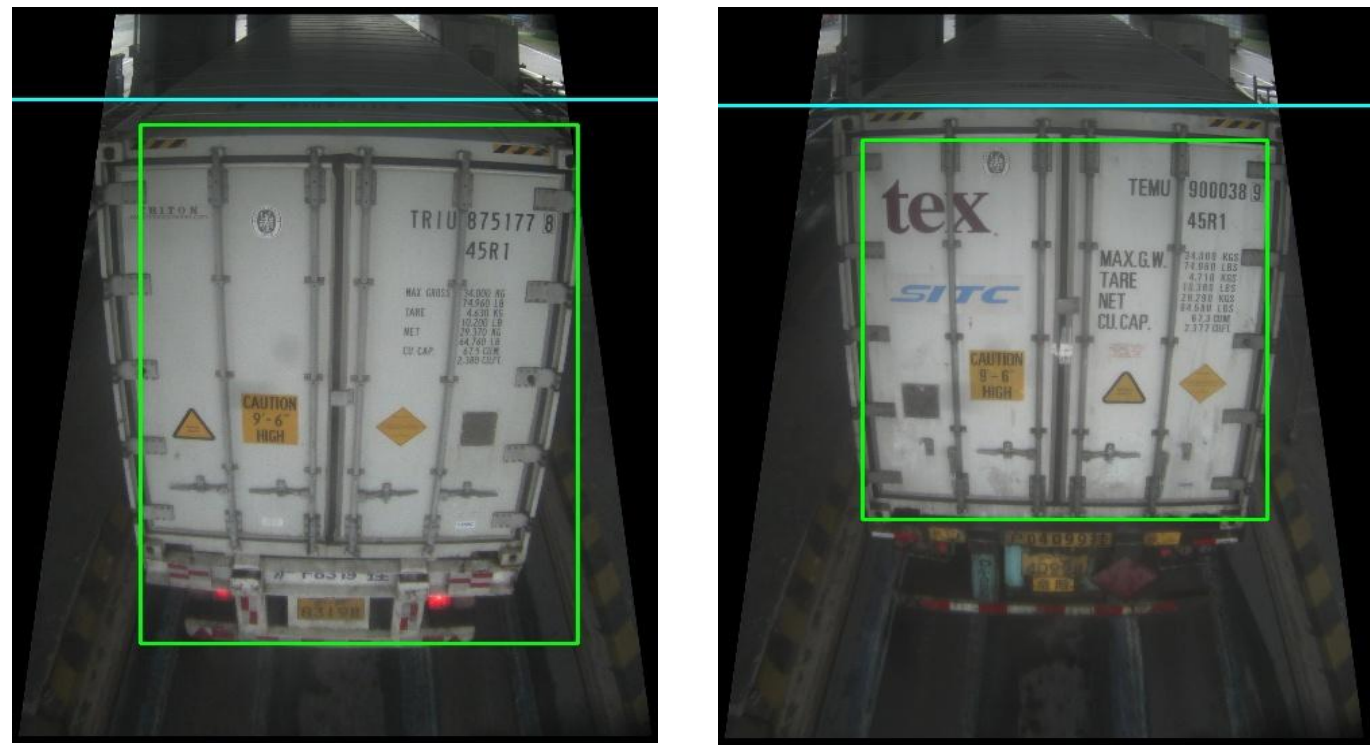

\subsection{The Result Analysis}

We totally use 93 container images for testing, including 71 color images and 22 white images. 
Table 4.1 The test result of the method

\begin{tabular}{|l|l|}
\hline Total number & 93 \\
\hline The number of color containers & 71 \\
\hline The correct number of color container results & 70 \\
\hline The accuracy rate of the extraction of color containers & $98.6 \%$ \\
\hline The number of white containers & 22 \\
\hline The correct number of white container results & 21 \\
\hline The accuracy rate of the extraction of color containers & $95.4 \%$ \\
\hline The overall accuracy rate & $\mathbf{9 7 . 8 \%}$ \\
\hline
\end{tabular}

\section{Conclusion and Prospect}

With the rapid development of container transportation, the automatic container character recognition system becomes very important, and the detection of container body is a very important step in the system.

In this paper, we have proposed a detection method for the container body base on HSV color model and texture features. The method is mainly for solving the problem that traditional method of background subtraction can't detect the container body effectively. Because of the obvious color feature of the color container, we use HSV color model for the detection, and considering that the color feature of the white container body is not very obvious, we combine the texture features for detection. The traditional method is very sensitive to the brightness changes of the background, however, the method we have proposed don't rely on the background, and has better robustness.

Due to time constraints, the method still has a lot of shortcomings. The main problem is the image with strong light irradiation and the image of night. Because there are a lot of disturbances in the image, the detection effect of our method is not very satisfactory. If those images can be pretreated by proper methods to adjust the light irradiation, the detection result of the container body will be more satisfactory, and this will be a very important step to make the container character recognition system a more practical system, and that is a direction for the future research.

\section{References}

1. Elgammal A, Hanvood D, Davis S. Nonparametric model for background subtraction [A]. In Proceedings of European Conference on Computer Vision[C]. Dublin, Ireland, 2000: 751-767.

2. Hu T. A Research of Container Character Recognition Based on Neural Network. The Dissertation of Wuhan Technological University. Wuhan, China, 2012.

3. Li X P, Yan Y. Analysis and Comparison of Background Modeling Methods. The 13th National Conference on Image and Graphics. Beijing, China, 2006.

4. Li Y, Sun Z X. An Improved Method for Motion Detection by Frame Difference and Background Subtraction. Journal of Image and Graphics. Nanjing, China, 2009.

5. Paragios N, Deriche R. Geodesic active contours and level sets for the detection and tracking of moving objects [J]. IEEE Transactions on Pattern Analysis and Machine Interface, 2000, 22(3): 266-280.

6. Stauffer C, Grinson W E L. Adaptive background mixture models for real-time tracking [A]. In Proceedings of IEEE Conference on Computer Vision and Pattern Recognition [C], Fort Collins, Colorado, USA, 1999: 246-252.

7. W ren C, A zarhayejani A, Darrell T, Pentland A P. Pfinder: real-time tracking of the human body [J]. IEEE Transactions on Pattern Analysis and Machine Intelligence, 1997, 19(7): 780-785. 